\title{
Asymptomatic Posterior Synovial Cyst of the Hip: A Case
}

\section{Report}

\author{
Nassar L ${ }^{1}$, Najem E1, Moukaddam $\mathrm{H}^{1}$, Farah $\mathrm{N}^{2}$ and Berjawi G1 ${ }^{1}$ \\ ${ }^{1}$ Diagnostic Radiology Department, American University of Beirut Medical Center, \\ Lebanon \\ ${ }^{2}$ Orthopedic Surgery Department, Bellevue Medical Center, Lebanon \\ *Corresponding author: Ghina Berjawi, Diagnostic Radiology Department,
}

\section{Case Report}

Volume 3 Issue 3

Received Date: May 31, 2019

Published Date: June 28, 2019

DOI: $10.23880 /$ crij-16000148

American University of Beirut Medical Center, Cairo Street, Beirut P0 Box 11-0236, Lebanon, Tel: +9613373708; Email: gb02@aub.edu.lb

\section{Abstract}

Synovial and ganglion cysts are lesions that can arise around various bodily joints. Cystic lesions of the hip are rare and may present with a wide spectrum of clinical signs and symptoms that are mostly due to compression of nearby nerve and vessels. In this paper, we present the first case of a 46-year-old previously healthy female with an incidental asymptomatic synovial cyst arising from the posterior aspect of the hip joint coursing in the vicinity of the sciatic nerve, bordering it in its pelvic segment and mimicking an adnexal mass on ultrasound.

Keywords: Synovial Cyst; Ganglion Cyst; Paralabral Cyst; Pelvic Cyst

Abbreviations: SC: Synovial cysts; GC: Ganglion cysts; MRI: Magnetic Resonance Imaging; PC: Paralabral cysts; US: Ultrasound.

\section{Introduction}

Synovial cysts (SC) and ganglion cysts (GC) are lesions that can arise around various bodily joints [1]. SC are characterized by a synovial lining and are filled with synovial fluid whereas GC are lined with connective tissue, lack a synovial lining and are thought to result from a myxomatous tissue degeneration [1]. However, SC and GS are radiologically indistinguishable and are hence used synonymously [2]. Cystic lesions of the hip are relatively rare and tend to be asymptomatic in up to $26 \%$ of the cases [1]. However, they may present with a wide range of clinical signs and symptoms that are mostly due to compression of nearby nerves and vessels. Limited cases have described a retroperitoneal extension of SC with compression of the colon, urinary bladder or ureter [3]. In this paper, we present the first case of a 46-yearold previously healthy female with an incidental asymptomatic synovial cyst arising from the posterior aspect of the hip joint coursing in the vicinity of the sciatic nerve, bordering it in its pelvic segment mimicking an adnexal mass on ultrasound.

\section{Case Presentation}

A 46-year-old previously healthy woman presented to her gynecologist for a routine visit. Physical examination was normal with a negative review of system including no groin, thigh or back pain, no lower limb edema, radicular pain, urinary symptoms or decreased peripheral pulses. A pelvic screening ultrasound was performed upon the patient's request and showed a cystic lesion in the right aspect of the pelvis, unrelated to the ovary (images not shown). MRI was done for a better characterization and 


\section{Clinical Radiology \& Imaging Journal}

showed a lobulated circumscribed lesion with hypointense signal on T1, hyperintense signal on T2 and no internal enhancement after gadolinium administration indicating a cystic nature (Figures 1a \& $1 \mathrm{~b}$ ).

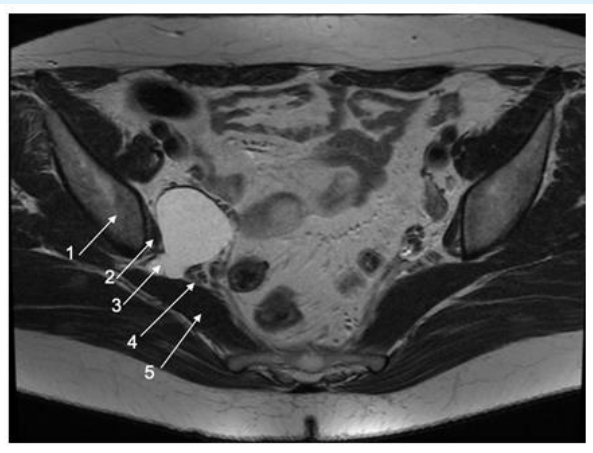

$1 \mathrm{a}$

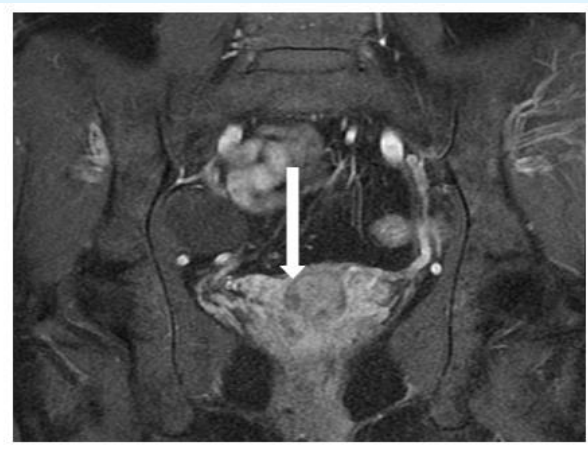

$1 \mathrm{~b}$

Annotations: 1-iliac bone, 2-obturator internus muscle, 3-synovial cyst, 4-sciatic nerve, 5-piriformis muscle.

Figures 1(a \& b): Axial T2 weighted image 1a shows a homogeneous hyperintense signal of the lesion. Coronal T1 image after fat saturation and administration of gadolinium shows no enhancement in $1 \mathrm{~b}$.

The lesion arises from the posterior aspect of the right femoroacetabular joint, courses superiorly for about $4 \mathrm{~cm}$ underneath the gemellus superior muscle. It then exits between the obturator internus and piriformis muscles into the pelvis, forming a $4.3 \times 3.2 \mathrm{~cm}$ cystic mass detected on ultrasound overlying the right ovary and abutting the sciatic nerve in its pelvic portion. Of note, there is a small communication between the cystic lesion and the posterior joint capsule (Figures 2a-2c). No further investigations or therapeutic procedures were done since the patient was asymptomatic and the lesion was clearly benign. The patient was informed and advised to followup in case of development any pelvic, thigh or back pain or neurological symptoms.

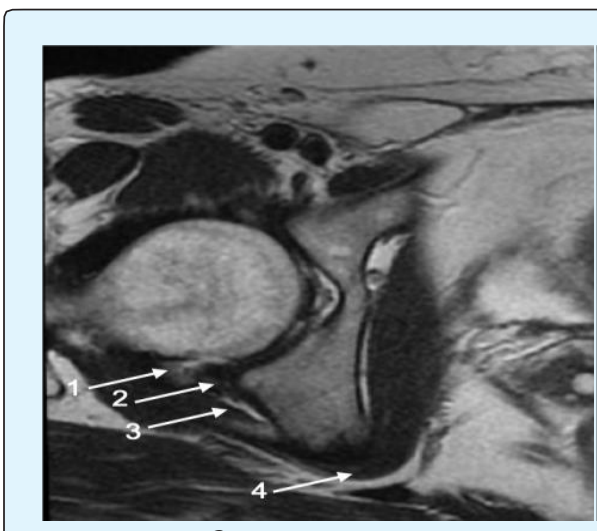

$2 \mathrm{a}$

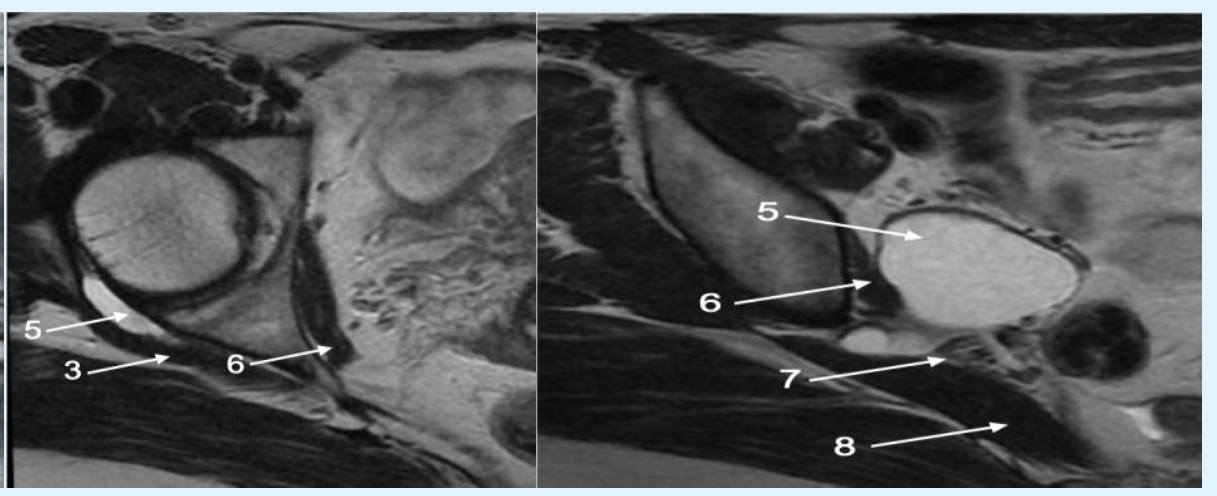

$2 \mathrm{~b}$ 2c

Annotations: 1-origin of the synovial cyst, 2-posterior labrum, 3-superior gemellus muscle, 4-obturator internus tendon, 5-synovial cyst, 6-obturator internus muscle, 7-sciatic nerve, 8-piriformis muscle.

Figures 2(a-c): Cropped T2 weighted axial images at the level of the right hip joint. The lesion arises from the posterior aspect of the right femoroacetabular joint (arrow-2a), travels underneath the gemellus superior muscle (arrow-2b), then exists into the pelvis between the piriformis muscle and obturator internus (2c). 


\section{Clinical Radiology \& Imaging Journal}

\section{Discussion}

\section{Cystic Lesions of the Hip Joint}

SC and GC are frequent benign cystic lesions occurring in various bodily joints. They are usually associated with degenerative joint disease, rheumatoid arthritis, trauma and tumors [3,4]. Angelini A, et al. have analyzed previous reported cases of anterior SC and GC of the hip and showed that out of 39 reported cases, $44 \%$ had no comorbidities while $31 \%$ had rheumatoid arthritis, $23 \%$ had osteoarthritis and $2 \%$ had hip contractures [3].

In the hip joint, cystic lesions are more likely to consist of SC rather than GC. Indeed, the knee is the most common joint followed by the hip where SC is more common than GC. GC occurs primarily in the wrist arising most commonly from the dorsal aspect of the scapholunate ligament followed by the radioscaphoidscapholunated interval, the scaphotrapezial, or the metacarpotrapezial joints [4]. Paralabral cysts (PC) are common cystic lesions in the hip joint and are associated with an intraarticular hip pathology, in particular labral tears [5]. According to Magerkurth 0, et al. paralabral cysts occur in up to $80 \%$ in the anterior hip and only $17 \%$ in the posterior hip with anterosuperior location being the most common $[5,6]$. Indeed, PC is often described on MRI as round or oval with fluid like signal multiloculated lesion averaging $11 \mathrm{~mm}$ in size [6]. Paralabral cysts of the hip are highly associated with full-thickness labral tear involving the base of the labrum at the transitional zone [6]. Although, few cases may occur in the absence of labral abnormality, the absence of a labral tear should generally raise the suspicion of SC, GC or atypical synovitis or tumor [6].

\section{Clinical Presentation and Management}

Cystic lesions around the hip are often asymptomatic. However, patients may present with pain, limited joint mobility, and signs of compression of the bowel, urinary tract, bladder, external iliac vessels, femoral vessels and femoral nerve. Limited cases have described a retroperitoneal extension of SC with compression of the colon, urinary bladder or ureter [3]. Rare cases have documented compression of the sciatic nerve by cystic lesions of the hip. Indeed, Juglard, et al. was the first to report in 1991 a case of sciatic nerve compression by a GS. Crawford 0, et al. has then reported three cases with sciatic nerve compression by a SC in post-operative patients following total hip replacement [1].

Sciatica is most commonly caused by disc herniation or spinal stenosis with extraspinal etiologies being less frequent $[7,8]$. Extraspinal etiologies include heterotopic ossification, sacroiliitis, angiosarcoma of the sacrum, or piriformis syndrome and cysts [7]. To date, only four cases of extraspinal sciatica caused by cystic lesions have been reported in the literature [7]. A first case reports describes a 51-year-old male patient presenting with increasing right buttock pain radiating downward to the posterior thigh and calf. An MRI revealed a multilocular cystic mass with high T2 WI and low T1 WI intensity arising from the postero inferior acetabular capsule coursing into the posterior aspect of the gemellus and obturator internus, compressing the sciatic nerve at the level of the sciatic notch [7]. Another case report documents a 48-year old woman with a 12 months history of intermittent pain in the groin and left buttock on exertion. The patient reported aggravating pain with radiation to the left lower limb.

MRI showed a $10 \mathrm{~cm}$ long ganglion cyst originating from the dorsal aspect of the hip joint. Interestingly, the mass accompanied the sciatic nerve along its course extending through the sciatic notch. An L5 root compression was noted in the intrapelvic segment of the cyst [8]. A third case describes a 63-year old man complaining of left buttock and thigh pain. An MRI of the pelvis showed a $5 \mathrm{~cm}$ cystic lesion which arose from the postero inferior labrum with compression of the sciatic nerve [9]. Interestingly all the described lesions have a similar course to the SC portrayed in our case. Indeed, they all arise from the posterior aspect of the acetabulum and course between the gemellus and obturator internus. The relationship to the sciatic nerve slightly varies between all the cases. Sciatica was only noted in cases of sciatic nerve compression.

Whereas asymptomatic cysts may be followed-up with no medical or surgical intervention, the treatment of cystic lesions of the hip depends on their size, severity of symptoms, nature of the underlying or associated condition, and the presence or absence of the local compression [1]. In fact, an enlarged or painful SC can be treated medically with non-steroidal anti-inflammatory drugs, application of local heat and physical therapy. However, if the cystic lesion is associated with rheumatoid arthritis, prednisone and methotrexate are recommended or injection of a local anesthetic and/or corticosteroids after aspiration of the cystic content [1]. In case of compressive symptoms, needle aspiration or surgery is advised [1].

\section{Radiological Features on Ultrasound and MRI}

Ultrasound (US) is widely used as a primary tool in the evaluation of suspected cystic lesions. In fact, it helps 


\section{Clinical Radiology \& Imaging Journal}

confirm the cystic nature of the lesion determines a possible communication between the cyst and joint cavity and assesses the nearby joints for the evaluation of associated disorders. SC and GC have different sonocompressibility with GC being harder to compress due to its thick content and dense fibrous capsule. Hence, sonocompressibility can help the operator differentiate between SC and GC. However, it is only applicable if the cystic lesion is palpable and fully covered by the probe [2]. MRI is used on the other hand for better characterization of cystic lesions because of its optimal soft tissue contrast, and its ability to differentiate cystic from solid lesions and detect small enhancing solid nodules or suspicious thickened septations [2].

Both SC and GC appear as well circumscribed lesions on MRI and may be lobulated with thin wall and thin septa along with internal or wall calcifications. As previously mentioned, they appear as hypo to iso-intense on T1 weighted images, homogeneously hyperintense on fluid sensitive sequences with only wall or thin septal enhancement. GS, due to their mucinous content may show however a higher signal on T1 and a lower signal on T2. Cysts complicated by either infection or hemorrhage may show increased wall thickness and internal heterogeneous hyperintensity on T1-WI and hypointense signal on T2-WI [2]. Identification of an intra-articular communication does not serve as an accurate radiological marker to differentiate between SC and GC. In fact, both lesions may or may not communicate with the joint space with less than $50 \%$ of the cases being detected on standard US and MRI [2,4]. MR arthrography with delayed imaging was found to be useful in the identification of a joint-cyst communication [2].

However, the topographic location remains the single most reliable criterion in differentiating between the two cystic lesions $[2,4]$. Indeed, GC were reported in up to $70 \%$ of soft tissue masses at the level of the wrist whereas SC were found to be the most common in the knee and hip joints [2,4]. Hence, the identified cystic lesion in our patient was hypothesized to be a SC rather than a GC.

\section{Conclusion}

Although only symptomatic when large and more likely in certain location, we present here a rare of the largest asymptomatic SC of the hip arising in a posterior location, extension into the pelvis through the sciatic notch mimicking an adnexal mass on US.

\section{References}

1. Yukata K, Nakai S, Goto T, Ikeda Y, Shimaoka Y, et al. (2015) Cystic lesion around the hip joint. World journal of orthopedics 6(9): 688-704.

2. Giard MC, Pineda C (2015) Ganglion cyst versus synovial cyst? Ultrasound characteristics through a review of the literature. Rheumatol int 35(4): 597605.

3. Angelini A, Zanotti G, Berizzi A, Staffa G, Piccinini E, et al. (2018) Synovial cysts of the hip. Acta Biomed 88(4): 483-490.

4. Neto N, Nunnes P (2016) Spectrum of MRI features of ganglion and synovial cysts. Insights imaging $7(2)$ : 179-186.

5. Scott E, Westermann R, Rosneck J (2018) A posterior labral cyst associated with an anterior labral tear of the hip. Skeletal radiology 47(6): 849-852.

6. Magerkurth O, Jacobson JA, Girish G, Brigido MK, Bedi A, et al. (2012) Paralabral cysts in the hip joint: findings at MR arthrography. Skeletal Radiol 41(10): 1279-1285.

7. Ravi M, Raja K, Asok P (2017) Acetabular paralabral cyst-A rare cause of sciatica. IJSR 6(4).

8. Briem T, Haemmerle G, Kramers-de QI, Leunig M (2016) Synovial Ganglion of the Hip as a Rare Cause of L5 Radiculopathy: A Case Report. JBJS Case Connect 6(3): 59.

9. Lakhotia D, Prashant K, Shon WY (2017) Ganglion cyst of the hip mimicking lumbar disk herniation-A case report. J Clin Orthop Trauma 8(2): 153-155. 